\title{
Design, Simulation and Fabrication of a New Terahertz Cross-shaped Metamaterial Bandpass Filter to Obtain a Narrow Frequency Bandwidth
}

hojatollah shahounvand ( $\sim$ hojazish515886@gmail.com )

Islamic Azad University of Arak https://orcid.org/0000-0002-5076-3095

Azim Fard

Islamic Azad University of Arak

Mohammad Bagher Tavakoli

Islamic Azad University of Arak

\section{Research Article}

Keywords: Terahertz metamaterial cross-shaped bandpass filter, Frequency width at half maximum (FWHM), Unwanted frequency, Transmission coefficient

Posted Date: August 11th, 2021

DOI: https://doi.org/10.21203/rs.3.rs-728494/v1

License: (c) (i) This work is licensed under a Creative Commons Attribution 4.0 International License. Read Full License

Version of Record: A version of this preprint was published at Optical and Quantum Electronics on January 12th, 2022. See the published version at https://doi.org/10.1007/s11082-021-03502-w. 


\title{
Design, simulation and fabrication of a new terahertz cross-shaped metamaterial bandpass filter to obtain a narrow frequency bandwidth
}

\author{
Hojatollah Shahounvand, Azim Fard and Mohammad Bagher Tavakoli
}

\begin{abstract}
In this study, a new terahertz cross-shaped metamaterial bandpass filter is designed, simulated and fabricated. It provides a very good transmission coefficient and much lower unwanted frequencies than other filters do. The simulation is conducted by the CST STUDIO software and the FDTD method.
\end{abstract}

Keywords: Terahertz metamaterial cross-shaped bandpass filter, Frequency width at half maximum (FWHM), Unwanted frequency, Transmission coefficient

\section{Introduction}

So far, many designs and methods have been presented to construct terahertz metamaterial bandpass filters with different aperture geometries. How these aperture geometries affect transmission properties has also been discussed. Terahertz cross-sectional metamaterials are intended for the selective transmission of terahertz waves along a desired wavelength or frequency range. The structures of these materials range from a simple periodic array of holes on a thin metal film to complex multilayer structures made through nano-fabrication techniques. In general, it is very important to have a pattern for the designing and development of terahertz bandpass metamaterials because it determines the transmission property of the peaks in terahertz spectra. A comprehensive overview of the designs for terahertz metamaterial bandpass filters is provided here [1-3].

Light transmission in the visible spectrum can be achieved through apertures smaller than the wavelength created in thin metal layers. It has been well established that light transmission through such structures constructed on metal films can be increased for several times. The physics of this phenomenon is elucidated, and it is believed that the Surface of Plasmon Polaritons (SPPs) produced by sub-wavelength structures serves for electromagnetic radiation. In recent years, researchers have successfully demonstrated that surface structures of appropriate characteristic wavelengths can have extraordinary transmissions in the terahertz field. Those structures have also found applications in such areas as terahertz measurement and imaging [1-5]

\section{Design basics}

Several factors must be carefully considered when designing an effective transient material in the terahertz domain. First, the resonant frequency of the terahertz transmission spectrum must be determined. To calculate that frequency for a typical aperture array diaphragm, researchers have developed an analytical method. It is based on certain points along the wavelength spectrum, such as the 
far infrared or terahertz region, where the size of the real and imaginary components of the dielectric constant of the metal is large. The following equation is used to determine the peak points in this case [1]:

$\lambda_{\text {peak }}=\frac{P}{\sqrt{i^{2}+j^{2}}} n_{s p}=\frac{P}{\sqrt{i^{2}+j^{2}}} \sqrt{\epsilon_{d}}$

Where $\lambda_{\text {peak }}$ is the central wavelength; the resonant frequency can be obtained by dividing the speed of light by the central wavelength. The other parameters in the equation are $\mathrm{P}$ as the periodic period, $n_{s p}$ as the refractive index of the surface, and $\epsilon_{d}$ as the surface dielectric constant. In addition to the transmission resonance for a typical aperture array diaphragm, the central terahertz transmission wavelength must be calculated for the other apertures. An experimental equation has been proposed to calculate terahertz bandpass cross-shaped apertures [1]. Müller et al. also proposed the following experimental equation to determine approximate central wavelengths [1-5]:

$\lambda_{\text {peak }}=1.8 L-1.35 W+0.2 P$

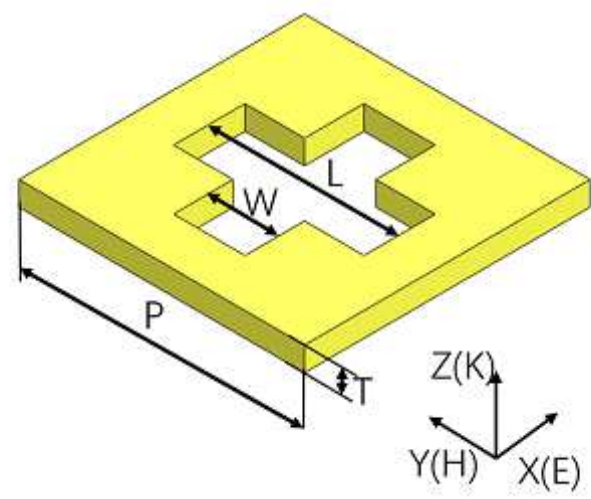

Figure 1. Schematic diagram of a THz bandpass filter with a cross-shaped aperture: the parameters L, W, $\mathrm{P}$ and $\mathrm{T}$ represent the arm length, arm width, period and thickness of the cross structure respectively[5]

Where $\mathrm{P}$ is the period, $\mathrm{L}$ is the width, and $\mathrm{W}$ is the length of the cross-shaped aperture. Those researchers also summarized several experimental equations with different coefficients for $\mathrm{L}, \mathrm{W}$ and $\mathrm{P}$, as compared to Equation (2). However, Equation (2) has been used more in recent works in which terahertz crossshaped metamaterials are constructed [4-6].

\section{Geometric pattern design}

The position of the transmission peak is a key feature for the best terahertz transmission and is considered the most important factor when designing terahertz SSPs. Using Equations (1) and (2), the geometric parameters can be changed to effectively determine the location of the transmission peaks. Therefore, the basic design principles for terahertz bandpass metamaterials can be summarized as follows:

1) Select the appropriate aperture geometry according to the design needs. 
2) Determine the appropriate dimensional parameters to achieve the desired transmission property (e.g., location of the resonant frequency).

Based on these principles, researchers have proposed different geometric designs, such as different hole diaphragm geometries. These geometries include alternating circular or square perforated diaphragms, cross-shaped diaphragms, or slit rings, as shown in Figure 2. The following section provides a comprehensive review of diaphragm structures for terahertz bandpass metamaterials [1-4].

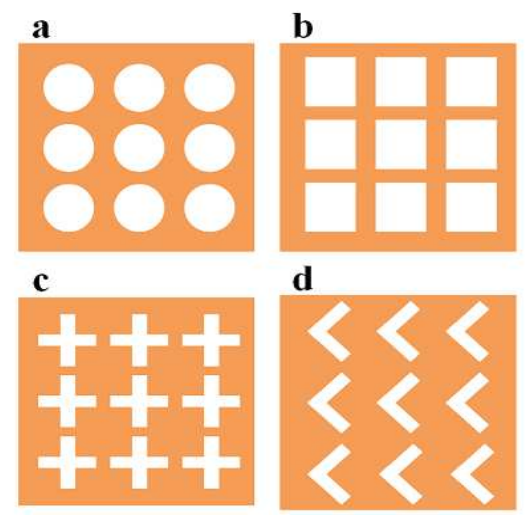

Figure 2. Different diaphragm geometries of terahertz bandpass metamaterials: a) circular, b) square, c) cross-shaped, and d) folding slit [1-4]

Circular diaphragms have been widely used for terahertz bandpass metamaterials. The research shows that the effect of a terahertz bandpass filter can be effectively achieved through a circular hole array. The effects of design factors on terahertz transmission have also been well studied. This includes the period, direction and diameter of holes. Experimental results show that, when these geometric parameters are changed, it is possible to obtain such terahertz transmission properties as the location of the resonant frequency, intensity of the resonance transfer and frequency width at half maximum (FWHM). The effect of square apertures on terahertz bandpass filters has also been investigated. Cao and Nahata mounted circular and square diaphragm arrays on 75-um stainless steel sheets and showed the importance of aperture shape in terahertz transmission with increasing resistance [1-3]. Geometry and aperture are important factors to consider when designing terahertz non-conducting materials. Appropriate geometric parameters must be selected to achieve the desired terahertz transmission property.

In addition to ordinary aperture arrays, innovative surface patterns have been designed and tested to achieve extraordinary terahertz transmission effects. In this regard, cross-diaphragm has been proposed and studied by many researchers in recent years [1-5].

A cross-shaped diaphragm is easily constructed, and it provides another important geometric pattern for the realization of terahertz resonance. Using various structural parameters, the resonance frequency of terahertz bandpass metamaterials can be modified [7]. Very narrow FWHM have been obtained using cross-shaped bandpass ultrastructures built into sources [ibid]. Table 1 shows the performance of three cross-shaped bandpass monolayer filters, as in Figure 1, achieved through simulation with the FDTD method. 
Another new type of structure is designed for the terahertz bandpass metamaterials in Jesus cross structure [8]. It has a transfer coefficient of $57 \%$ to $89 \%$, which is lower than that of a cross-shaped structure (Table 1). These types of structures have been simulated using the CST STUDIO software and compared with experimental results; they have proved to provide very similar results [8].

Table 1. Performance of three cross-shaped bandpass monolayer filters (Figure 1) obtained through FDTD simulation and THz-TDS system measurement [7]

\begin{tabular}{lllllll}
\hline Filter & $T$ & $T^{*}$ & $f_{c}(\mathrm{GHz})$ & $f_{c}^{*}(\mathrm{GHz})$ & $F W H M(\mathrm{GHz})$ & $F W H M^{*}(\mathrm{GHz})$ \\
\hline A & 0.9 & 1 & 557 & 550 & 154 & 177 \\
B & 0.89 & 0.98 & 372 & 367 & 70 & 74 \\
C & 0.75 & 0.91 & 325 & 325 & 53 & 56 \\
\hline
\end{tabular}

Another type of terahertz bandpass filters are produced through the design and fabrication of frequency selective surfaces (FSSs) that operate within a range of more than $300 \mathrm{GHz}$. These structures serve as open-space electromagnetic filters and, thus, provide multispectral remote sensing tools by dividing radiation into separate frequency channels [9]. This new design for quasi-optical filters has a drop of 0.3 $\mathrm{dB}$ at $700 \mathrm{GHz}$ and can be designed to be independent of the polarization of collision signals [9].

Of all the different geometries ever designed and fabricated for terahertz bandpass filters, cross-shaped bandpass filters are advantageous in terms of spectral transmission and frequency bandwidth [13-10].

\section{Filter structure and the results}

A cross-shaped bandpass filter is designed on a copper substrate using the CST STUDIO simulator software. The coordinates (p), (w) and (l) of every filter structure (Figure 1) are different at different frequencies. In the simulation process, to produce a filter with the output of a frequency spectrum near 2.2 terahertz, p, w and 1 have the values of 29, 100 and 154 micrometers respectively (Figure 1 and Equation 2). To confirm the accuracy of the simulation with the CST STUDIO software, these coordinates are evaluated against those reported in the studies conducted on cross-shaped filters [14-16].

In the next step, to reduce the frequency bandwidth of the output beam, two examples of this structure are placed at different distances (i.e. distance $d$ as in Figure 4). This causes the frequency bandwidth to differ from the initial state (single filter). The value of $d$ changes at the distances $\lambda, \lambda / 2$ and $\lambda / 4$ from the central wavelength (resonance wavelength). As observed, the maximum reduction in the frequency bandwidth is obtained in the $\lambda / 2$ mode, which is about half the frequency bandwidth of the initial state (single filter) (Figure 5) [5, 17-19].

According to the results obtained on the basis of Fabry-Pert principles, placing two filters in a row at the distance of half the wavelength reduces the frequency bandwidth. The results of this simulation are consistent with the computational results reported in some other sources $[13,20]$. 


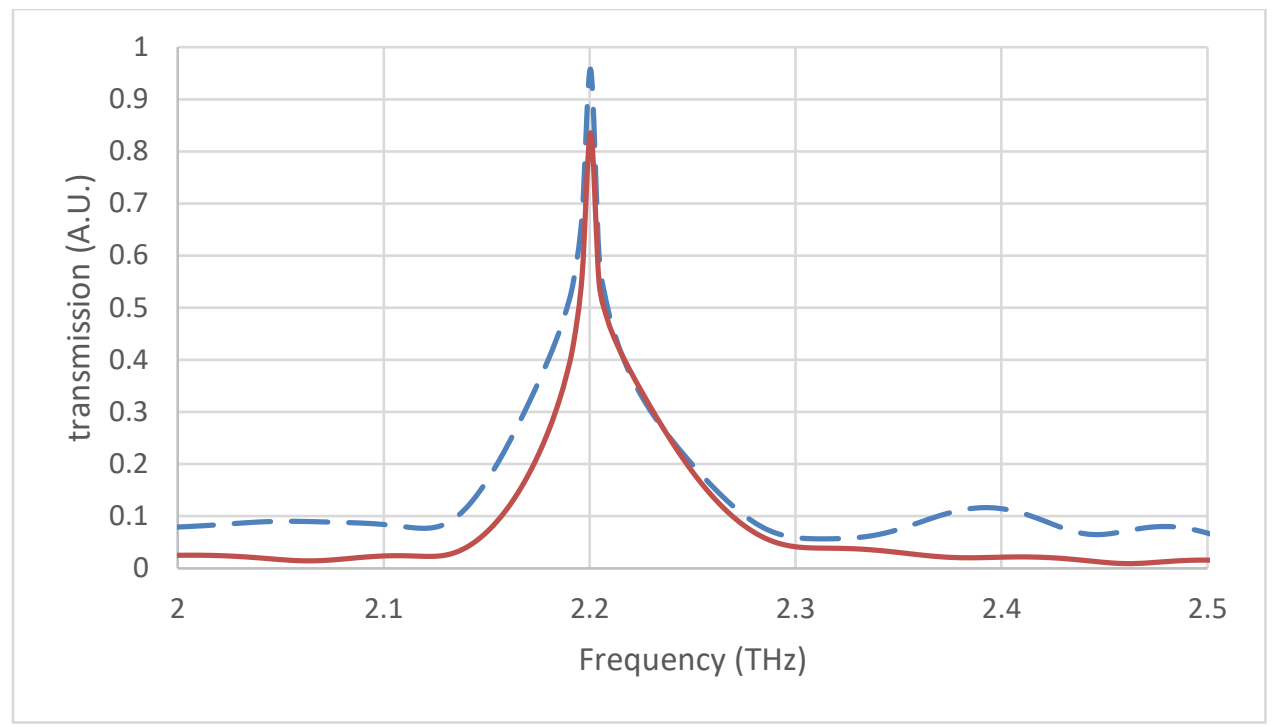

Figure 3. The output frequency of a cross-shaped filter at the central frequency of 2.2 terahertz with dimensions $\mathrm{L}=81, \mathrm{~W}=29$ and $\mathrm{P}=154 \mathrm{um}$

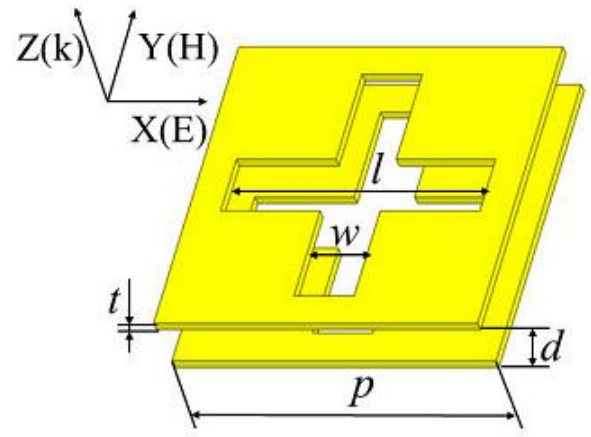

Figure 4. Two cross-shaped filters in a row at a distance of $d$ [5]

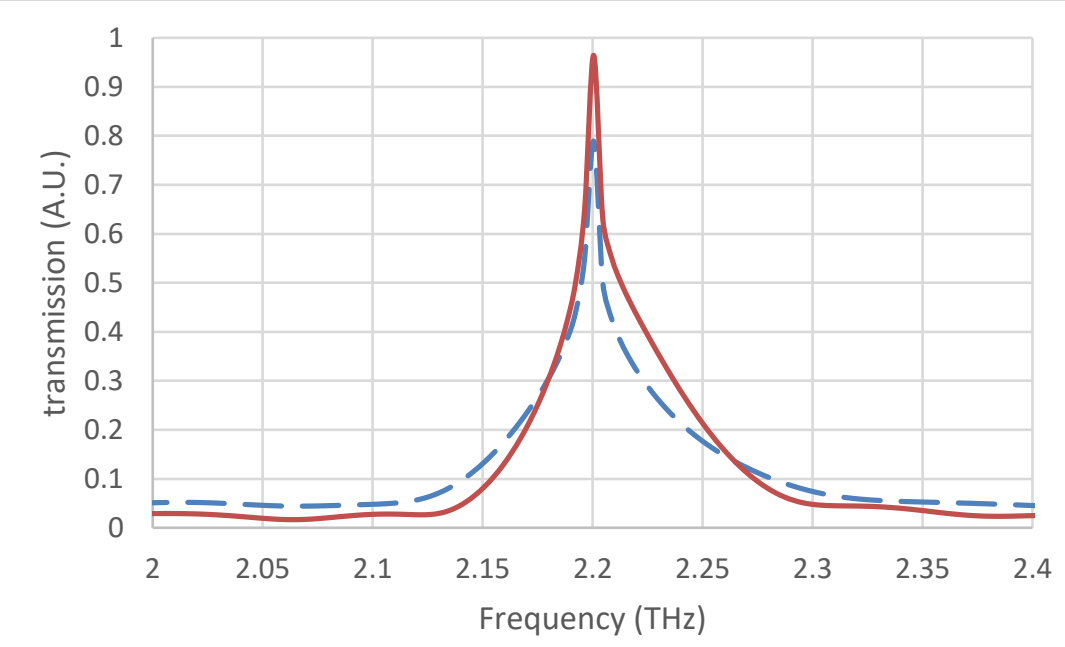


Figure 5. The bandwidth at a central frequency of 2.2 terahertz per $d=\lambda / 2$ for a single filter (dashedline diagram) and a dual filter in a row (dotted-line diagram)

\section{Proposing a new cross-shaped metamaterial bandpass filter}

In this section, a new cross-shaped bandpass filter is designed and simulated (Figure 6).
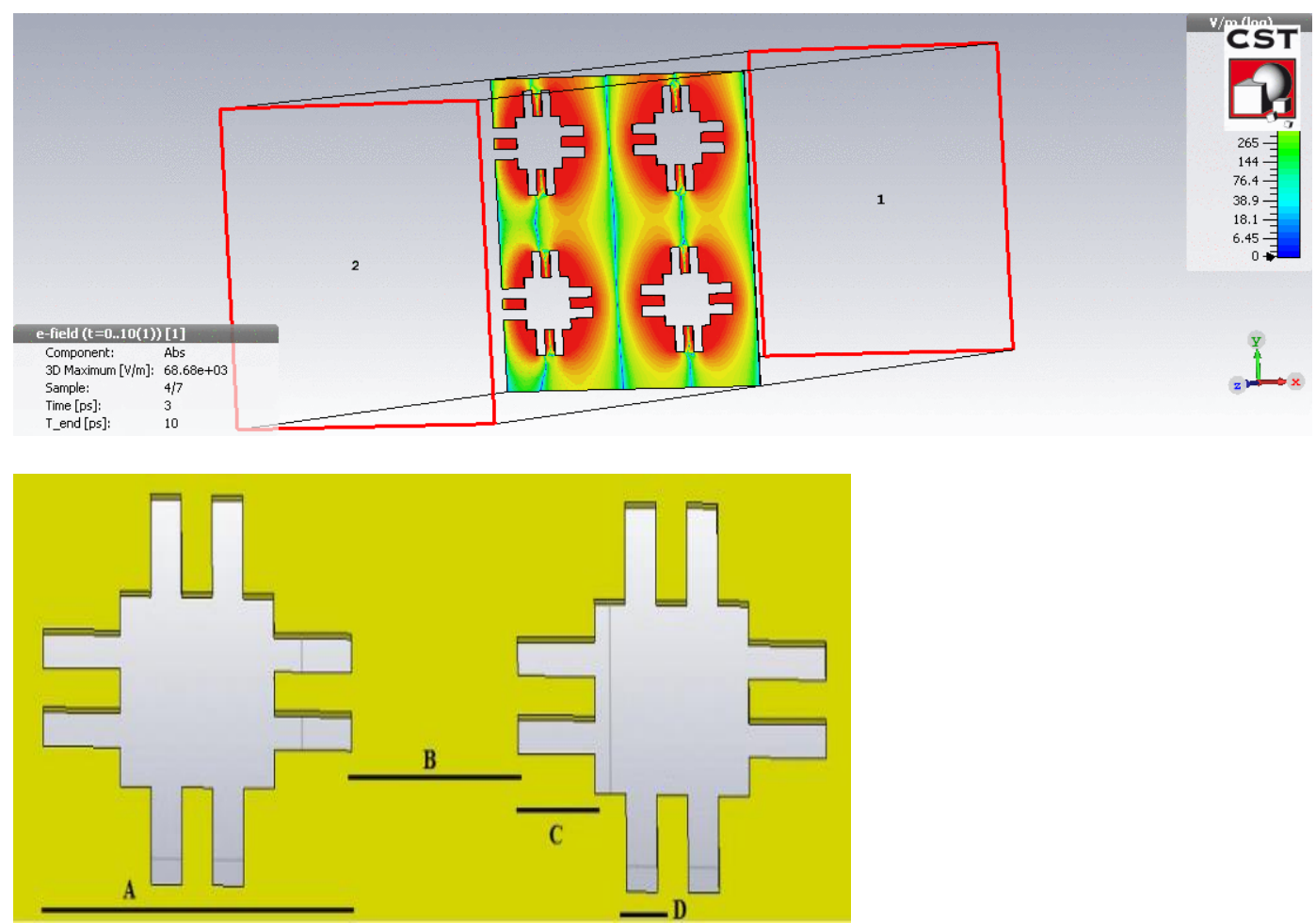

Figure 6. The proposed new cross-shaped bandpass filter (coordinates A, B, C and D are selected according to dimensions $\mathrm{L}, \mathrm{W}$ and $\mathrm{P}$ in Figure 1)

The A, B, C and D dimensions of the proposed cross-shaped bandpass filter are 154, 81, 29 and 9.6 micrometers respectively. These dimensions are used in accordance with the wavelength dimensions (Figure 1 and Equation 2) in cross-shaped filters $(A \equiv P, B \equiv L, C \equiv W, D \equiv 1 / 3 W$ ), which helps to compare the output of the proposal filter with that of cross-shaped ones. Through the simulation of crossshaped filters and the proposed filter, the outputs have been compared in terms of the percentage of transmission of the output spectrum, noise (unwanted frequency) (Figure 7) and FWHM (Figure 8). 


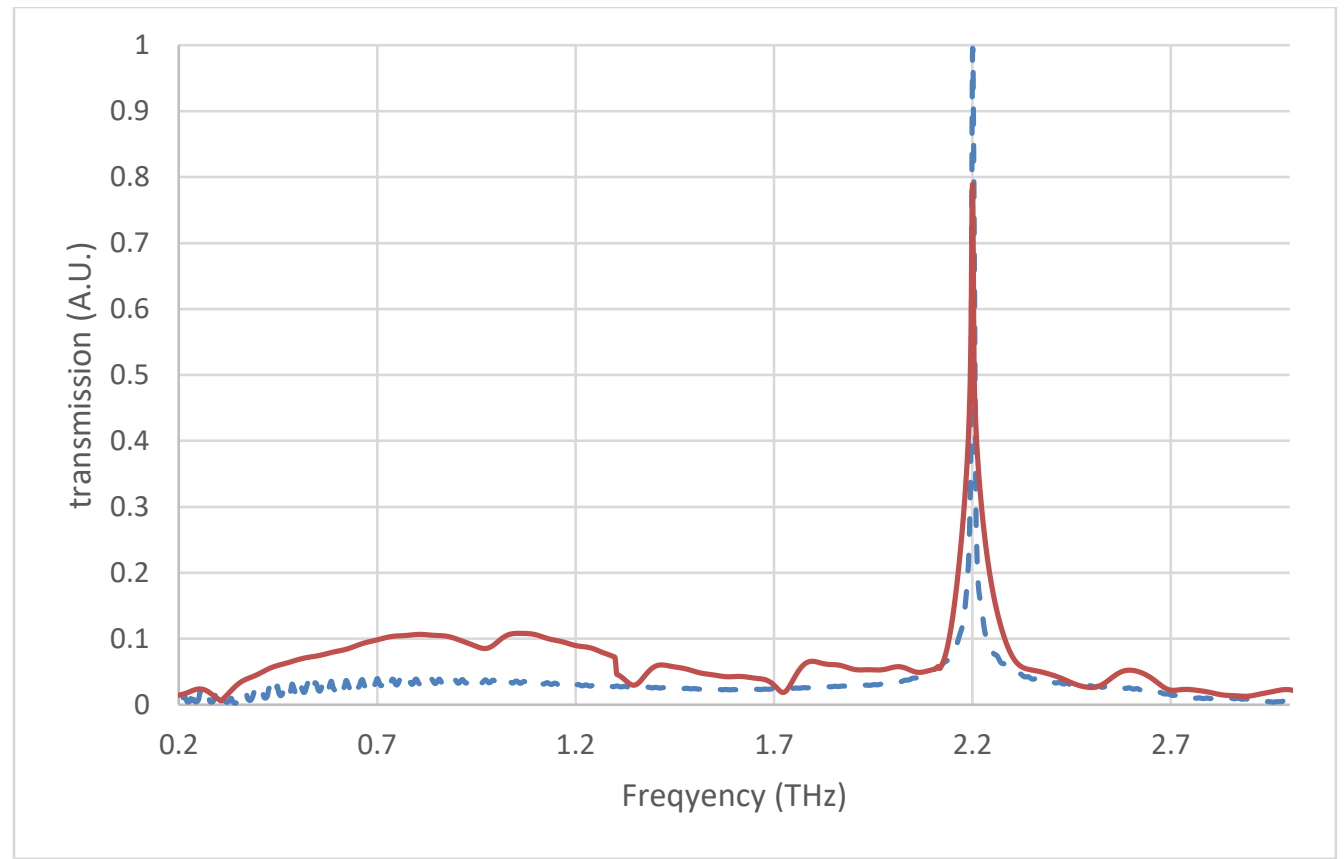

Figure 7. The effects of noise (unwanted frequencies) and the intensity of the output spectrum: a) the proposed filter (dotted-line diagram) and b) cross-shaped filter (dashed-line diagram)

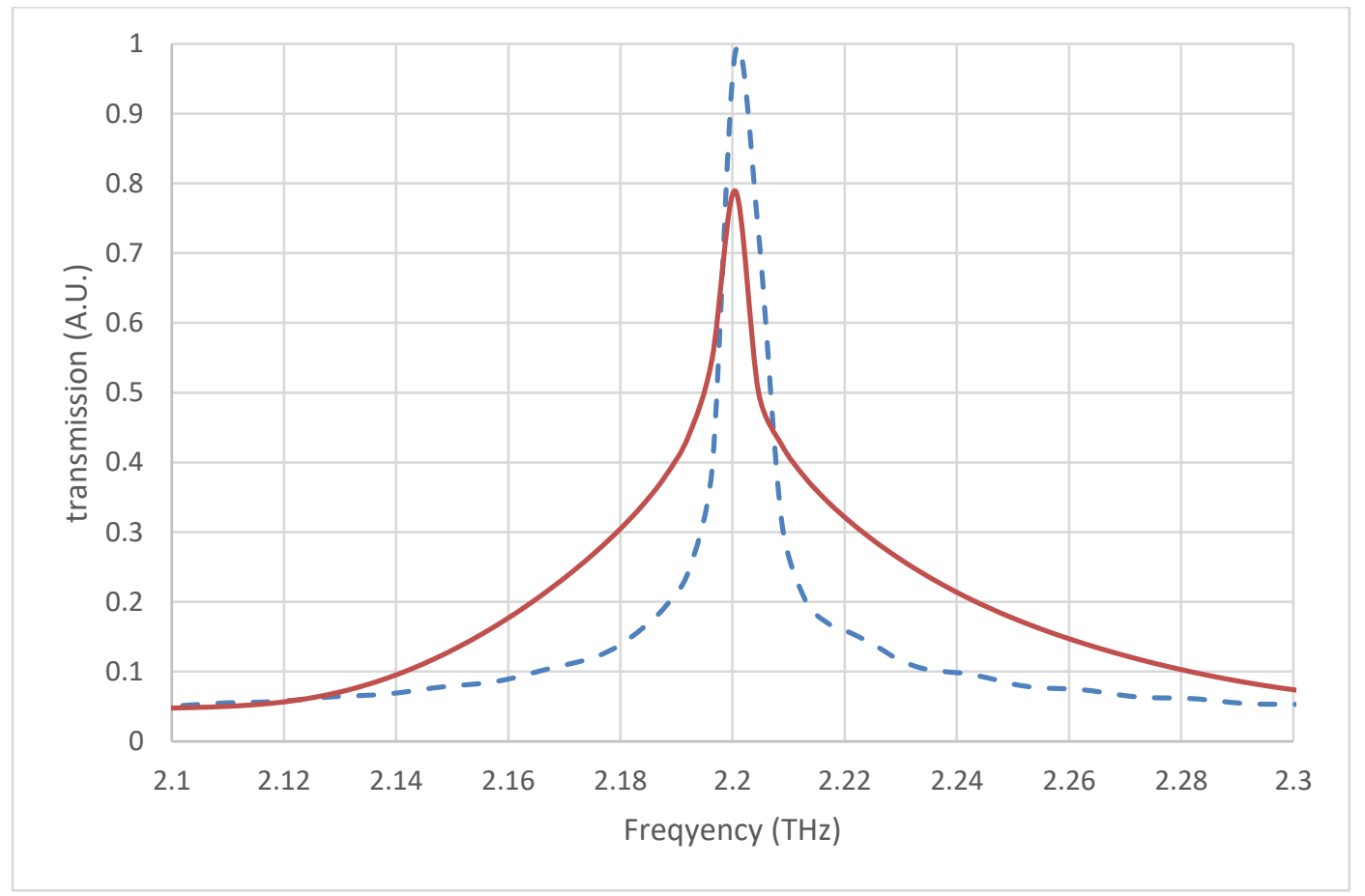

Figure 8 . The frequency bandwidths (FWHM) of a) the proposed filter (dotted-line diagram) and b) crossshaped filter (dashed-line diagram) 
Among the properties of the proposed filter, compared to built-in filters (especially cross-shaped filters), one may refer to higher output transfer intensity (of almost 100\%) and less noise effects (unwanted frequencies) in the whole range of 0.1 to 3 terahertz.

\section{Comparison of the fabricated experimental device and the simulated sample}

The transmission spectrum is measured by terahertz time domain spectroscopy (THz TDS) (Figure 9). The system uses a femtosecond laser with a wavelength of $780 \mathrm{~nm}$, a time width of less than 100 femtoseconds and an output power of $50 \mathrm{~mW}$. The laser is made by Taptica - Femto FErb780-Fibersmart. In this type of spectroscopy system, both antenna generators and detectors are made by Batop Company with the abbreviated code of PCA-90-01-10-800-h.

The built-in filter is shown in Figure 10. A substrate with a copper layer is used as a cathode in the electroplating process. The SU-8 photoresist is also deposited using a spin coater. Photolithography is the main process practiced with a UV exposure machine to make the graph of the mask projected onto the photoresist [18].

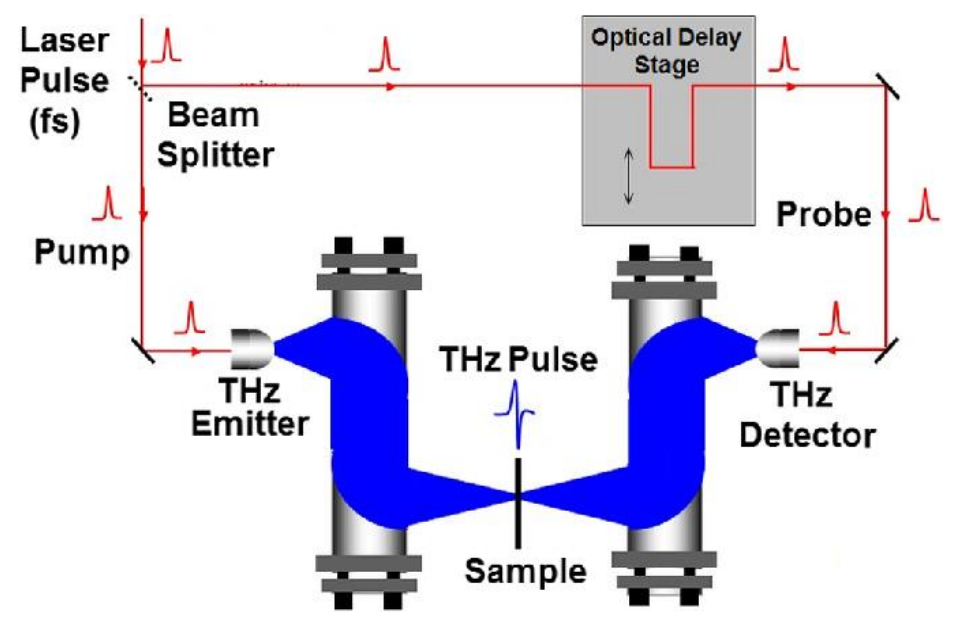

Figure 9. Schematic display of a terahertz time domain spectroscopy (THz TDS) system

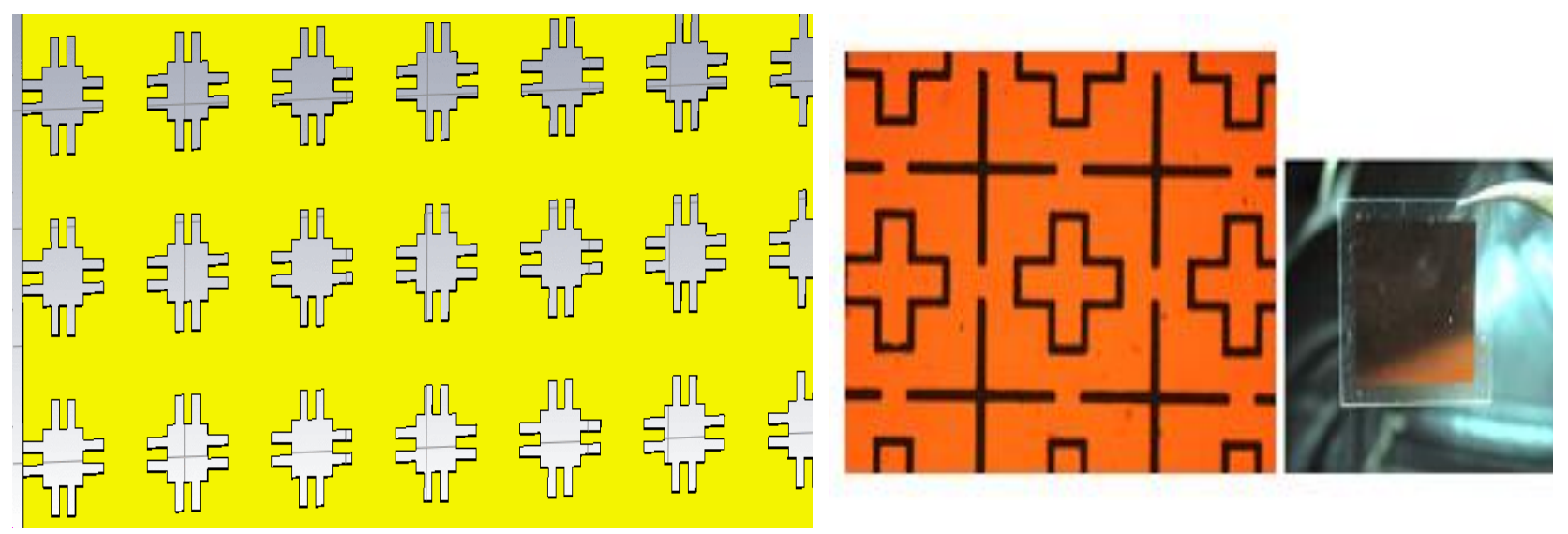

Finger 10. Image of the THz cross-shaped bandpass filters fabricated based on a photoresist [14]. 
Figure 11 shows two spectra of experimental results with THz-TDS system and simulation results with CST STUDIO software that are well matched.

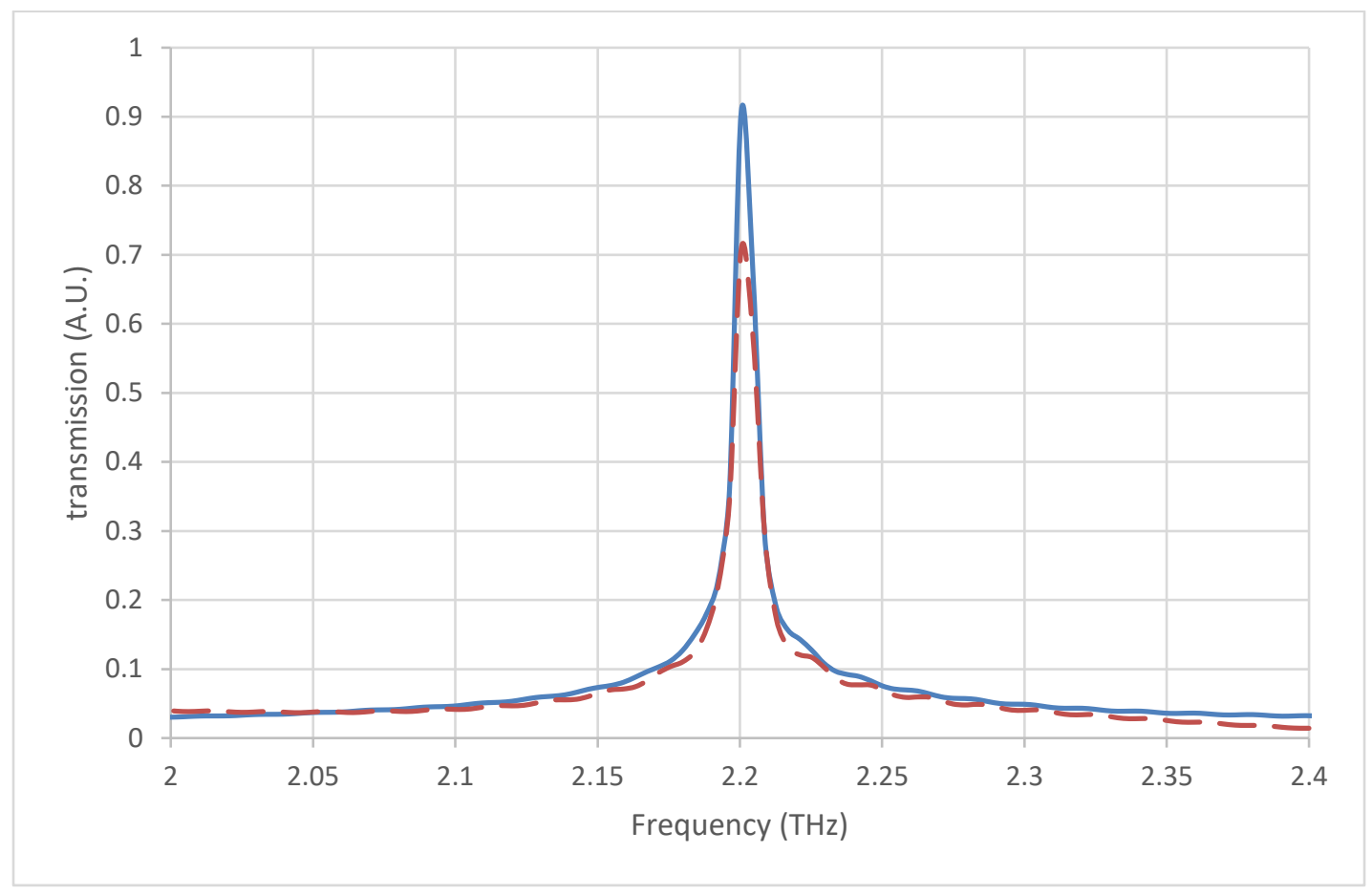

Figure 11. Comparison of the experimental results (dotted-line graph) with the simulation results (dashedline diagram)

\section{Conclusion}

A new bandpass filter is designed, simulated and fabricated based on the samples already made for terahertz cross-shaped bandpass filters. The output spectrum of the filter proves to be of a high percentage (approximately 100\%) and of an output noise (unwanted frequencies) less than the frequency range of 0.1 to 3 terahertz.

\section{References}

[1] Wang, Qinghua, et al. "Design, fabrication, and modulation of THz bandpass metamaterials." Laser \& Photonics Reviews 13.11 (2019): 1900071.

[2] Chiang, Yi-Ju, et al. "An ultrabroad terahertz bandpass filter based on multiple-resonance excitation of a composite metamaterial." Applied Physics Letters 99.19 (2011): 191909.

[3] Sedykh, E. A., V. Y. Soboleva, and M. K. Khodzitsky. "Tunable narrowband filters with cross-shaped resonators for THz frequency band." Journal of Physics: Conference Series. Vol. 643. No. 1. IOP Publishing, 2015.

[4] Paul, Oliver, René Beigang, and Marco Rahm. "Highly selective terahertz bandpass filters based on trapped mode excitation." Optics Express 17.21 (2009): 18590-18595. 
[5] Gao, Tao, et al. "Resonant Coupling Effects in a Double-Layer THz Bandpass Filter." Applied Sciences 10.15 (2020): 5030.

[6] Ao, Tianhong, et al. "Terahertz band-pass filters based on fishnet metamaterials fabricated on freestanding SiNx membrane." Optics Communications 405 (2017): 22-28.

[7] Lin, Yanzhang, et al. "Free-standing double-layer terahertz band-pass filters fabricated by femtosecond laser micro-machining." Optics express 25.21 (2017): 25125-25134.

[8] Xiong, Ri-Hui, and Jiu-sheng Li. "Terahertz transmission characteristics of free-standing fractal Jesuscross structure." Front. Phys. 8: 23. doi: 10.3389/fphy (2020).

[9] Dickie, Raymond, et al. "THz frequency selective surface filters for earth observation remote sensing instruments." IEEE Transactions on Terahertz Science and Technology 1.2 (2011): 450-461.

[10] Melo, Arline M., et al. "Cross-shaped terahertz metal mesh filters: historical review and results." Advances in Optical Technologies 2012 (2012).

[11] Ri-Hui, Xiong, and Li Jiu-Sheng. "Double-layer frequency selective surface for terahertz bandpass filter." Journal of Infrared, Millimeter, and Terahertz Waves 39.10 (2018): 1039-1046.

[12] Mittholiya, Kshitij, et al. "Cross-Shaped Rounded Corner Terahertz Bandpass Filter on Silicon."

[13] Shahounvand, Hojatollah, and Azim Fard. "Design and simulation of a new narrow terahertz bandpass filter." SN Applied Sciences 2.11 (2020): 1-7.

[14] Yan, Zhendong, et al. "Perfect Absorption and Refractive-Index Sensing by Metasurfaces Composed of Cross-Shaped Hole Arrays in Metal Substrate." Nanomaterials 11.1 (2021): 63.

[15] Liu, Dejun, et al. "Quasibound states in the continuum in terahertz free-standing metal complementary periodic cross-shaped resonators." arXiv preprint arXiv:2007.08104 (2020).

[16] Komarov, Vyacheslav V., and Valery P. Meschanov. "Transmission properties of metal mesh filters at 90 GHz." Journal of Computational Electronics 18.2 (2019): 696-704.

[17] Soboleva, V. Yu, et al. "Development of narrow bandpass filters based on cross cavities for the terahertz frequency range." Journal of Optical Technology 84.8 (2017): 521-524.

[18] Lu, Changgui, et al. "A new fold-cross metal mesh filter for suppressing side lobe leakage in terahertz region." Optics Communications 413 (2018): 190-195.

[19] Huang, Renzhi, et al. "Varactor-tunable Bandpass Frequency Selective Surface with Wide Tuning Range." 2020 9th Asia-Pacific Conference on Antennas and Propagation (APCAP). IEEE, 2020.

[20] Asgari, Somayyeh, and Tapio Fabritius. "Tunable mid-Infrared graphene plasmonic cross-shaped resonator for demultiplexing application." Applied Sciences 10.3 (2020): 1193. 